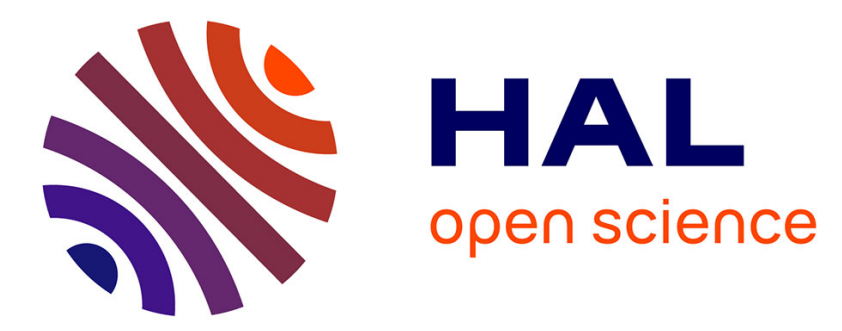

\title{
MR Diffusion and Perfusion Parameters: Relationship to Metabolites in Acute Ischaemic Stroke.
}

Vera Cvoro, Ian Marshall, Paul A Armitage, Mark Bastin, Trevor Carpenter, Carly Rivers, Martin Dennis, Joanna Wardlaw

\section{- To cite this version:}

Vera Cvoro, Ian Marshall, Paul A Armitage, Mark Bastin, Trevor Carpenter, et al.. MR Diffusion and Perfusion Parameters: Relationship to Metabolites in Acute Ischaemic Stroke.. Journal of Neurology, Neurosurgery and Psychiatry, 2010, 81 (2), pp.185. 10.1136/jnnp.2008.168393 . hal-00552748

\section{HAL Id: hal-00552748 \\ https://hal.science/hal-00552748}

Submitted on 6 Jan 2011

HAL is a multi-disciplinary open access archive for the deposit and dissemination of scientific research documents, whether they are published or not. The documents may come from teaching and research institutions in France or abroad, or from public or private research centers.
L'archive ouverte pluridisciplinaire HAL, est destinée au dépôt et à la diffusion de documents scientifiques de niveau recherche, publiés ou non, émanant des établissements d'enseignement et de recherche français ou étrangers, des laboratoires publics ou privés. 
MR DIFFUSION AND PERFUSION PARAMETERS: RELATIONSHIP TO METABOLITES IN ACUTE ISCHAEMIC STROKE.

\author{
Authors: Vera Cvoro, MRCP \\ Ian Marshall, ${ }^{\mathrm{a}} \mathrm{PhD}$ \\ Paul A Armitage PhD \\ Mark E Bastin ${ }^{\mathrm{a}}$ DPhil \\ Trevor Carpenter, $\mathrm{PhD}$ \\ Carly S Rivers, ${ }^{\mathrm{b}} \mathrm{PhD}$ \\ Martin S Dennis MD FRCP (ed) \\ Joanna M Wardlaw, MD, FRCR, FRCP, FMedSci
}

Address: Divisions of Clinical Neurosciences and ${ }^{\mathrm{a}}$ Medical Physics, University of Edinburgh, Western General Hospital, Crewe Rd, Edinburgh, EH4 2XU; ${ }^{\text {b Clinical Trials }}$ Research Unit, University of Leeds, Leeds, UK.

Corresponding author: Prof. Joanna M. Wardlaw, SFC Brain Imaging Research Centre, Division of Clinical Neuroscience, Western General Hospital, Crewe Rd, Edinburgh, EH4 2XU, UK. Tel: +44 131537 2943; Fax: +44 $131 \quad 332$ 5150; Email: joanna.wardlaw@ed.ac.uk

Key words: acute ischemic stroke, diffusion imaging, magnetic resonance imaging, penumbra, perfusion imaging, spectroscopy.

Word count: 3950

Abstract 228 words

References 43

Tables 1 
JNNP 2008/168393 revision 3, Cvoro et al

Figures 2 (1 colour) 
JNNP 2008/168393 revision 3, Cvoro et al

\section{ABSTRACT}

Background: MR diffusion and perfusion imaging are used to identify ischemic penumbra, but there are few comparisons with neuronal loss and ischaemia in vivo. We compared N-acetyl aspartate (NAA, found in intact neurons) and lactate (anaerobic metabolism) with diffusion/perfusion parameters.

Methods: We prospectively recruited patients with acute ischemic stroke and performed MR diffusion tensor, perfusion (PWI) and proton chemical shift spectroscopic imaging (CSI). We superimposed a $0.5 \mathrm{~cm}$ voxel grid on the diffusion-weighted images (DWI) and classified voxels as 'definitely abnormal', 'possibly abnormal', or normal on DWI appearance, and 'mismatch' for voxels in DWI/PWI mismatch areas. We compared metabolite (NAA, lactate), perfusion and ADC values in each voxel type.

Results: NAA differentiated 'definitely' from 'possibly abnormal', and 'possibly abnormal' from 'mismatch' (both comparisons $\mathrm{p}<0.01$ ) voxels, but not 'mismatch' from 'normal' voxels. Lactate was highest in 'definitely abnormal', and progressively lower in 'possibly abnormal', 'mismatch', then 'normal' voxels (all differences $\mathrm{p}<0.01$ ). There was no correlation between NAA and ADC or PWI values, but high lactate correlated with low ADC (Spearman $\rho=-0.41, p=0.02)$ and prolonged MTT (Spearman $\rho=0.42, p=0.02)$.

Conclusion: ADC and MTT indicate the presence of ischemia (lactate) but not cumulative total neuronal damage (NAA) in acute ischemic stroke, suggesting that caution is required if using $\mathrm{ADC}$ and PWI parameters to differentiate salvageable from non-salvageable tissue. Further refinement of the DWI/PWI concept is required prior to more widespread use. 
JNNP 2008/168393 revision 3, Cvoro et al

\section{INTRODUCTION}

In acute ischemic stroke, the ischemic penumbra is considered to be hypoperfused tissue where neurons are functionally silent but retain structural integrity and are potentially salvageable if blood flow can be restored quickly. The mismatch between magnetic resonance (MR) diffusion-weighted (DWI) and perfusion imaging (PWI) may be one way of identifying penumbra, but this approach requires standardisation. ${ }^{1-3}$ No consistent perfusion threshold has so far distinguished dead from salvageable tissue reliably. ${ }^{4-7}$ Several apparent diffusion coefficient (ADC) thresholds have been reported for salvageable/non-salvageable tissue ${ }^{8-10}$ but with considerable overlap between tissue that had already infarcted, was ischemic but viable, or normal but subsequently infarcted. ${ }^{11 ; 12}$ These studies all use the final infarct as shown on T2 or FLAIR imaging at several days to months after the stroke to determine early tissue viability thresholds. However many changes occurring in between the acute and late phases could influence the final tissue fate and may have added to the variability of acute DWI and PW threshold values.

An alternative method of determining early tissue viability would be to use proton MR spectroscopy (MRS). This detects N-acetyl aspartate (NAA) which, although its precise function is not clearly understood, is only found in intact functioning neurons in adults, is present in sufficient quantity to provide a clear measurable signal on MRS, and loss of NAA indicates neuronal death. ${ }^{13-15}$ Thus, NAA is reduced in infarcted tissue in patients. ${ }^{16 ; 17}$ In a permanent middle cerebral artery occlusion model, by six hours NAA had fallen to $50 \%$ of control with $30 \%$ of neurons appearing viable histologically; by 24 hours, NAA had fallen to $20 \%$ of control with few neurons appearing viable. ${ }^{18 ; 19}$ MRS also detects rapid rises in lactate due to anerobic metabolism in acute ischemic tissue. ${ }^{20 ; 21}$ If ADC or PWI directly indicate neuronal damage, then ADC and PWI values should correlate closely with NAA; if they mainly indicate ischemia, then ADC and PWI values 
JNNP 2008/168393 revision 3, Cvoro et al

should correlate closely with lactate rather than NAA. However there are few studies of MRS and DWI or PWI in acute ischaemic stroke to date. ${ }^{16 ; 21-27}$ While indicating that NAA fell and lactate rose in DWI-abnormal areas, these studies did not examine associations between metabolites, DWI and PWI parameters in detail across the whole stroke lesion. Many were unable to profile metabolite, diffusion and/or perfusion values because they used single voxel spectroscopy. ${ }^{16 ; 21 ; 25 ; 26}$ Many only sampled MRS and diffusion not perfusion. ${ }^{16 ; 21 ; 22 ; 25-27}$ Only one small study compared NAA with both DWI and PWI simultaneously in the DWI-visible lesion, but found very heterogeneous ADC values. ${ }^{24}$ Therefore we used proton MR spectroscopic imaging and a voxel based analysis to map NAA, lactate, DWI and PWI values across the acute ischaemic lesion and normal brain. 
JNNP 2008/168393 revision 3, Cvoro et al

\section{METHODS}

\section{Patient recruitment}

We prospectively recruited patients with symptoms of acute cortical ischemic stroke, without contraindications to MR imaging. An experienced physician obtained a detailed history and neurological examination, measured the stroke severity according to the National Institutes of Health Stroke Scale (NIHSS, http://www.strokecenter.org/trials/scales/), and determined the stroke type according to the Oxfordshire Community Stroke Project (OCSP) classification. ${ }^{28}$ Patients with lacunar stroke were excluded (lesion too small and usually no perfusion lesion) as well as those who were too ill to undergo MRI. The study was approved by the Local Research Ethics Committee, and subjects or their relatives gave written informed consent. All patients underwent usual investigations for stroke as well as the study procedures.

\section{MR imaging and MRS technique}

Patients underwent MR imaging, as soon as possible after stroke (but within a maximum of 24 hours) on a GE 1.5 T Signa LX (General Electric, Milwaukee, WI, USA) scanner. Imaging included axial $\mathrm{T}_{2}$-weighted fast spin echo $(\mathrm{T} 2 \mathrm{~W})$, axial diffusion tensor imaging (DTI) based on spin-echo echo-planar imaging (EPI), axial dynamic susceptibility contrast PWI using gradient-echo EPI, and single slice point resolved spectroscopy (PRESS) proton MRS chemical shift imaging (CSI). The imaging parameters for DTI were: field-ofview (FOV) $240 \times 240 \mathrm{~mm}, 15$ axial slices of thickness $5 \mathrm{~mm}$, slice gap $1 \mathrm{~mm}$, acquisition matrix $128 \times 128$, echo time (TE) $97.4 \mathrm{~ms}$ and repetition time (TR) 10 seconds. Diffusion sensitising gradients with scalar b-values of $1000 \mathrm{~s} / \mathrm{mm}^{2}$ were applied in six non-collinear directions. Perfusion imaging was performed using the dynamic signal change recorded up to 85 seconds following a bolus injection of a gadolinium-based contrast agent $(10 \mathrm{~mL}$ of 1 
JNNP 2008/168393 revision 3, Cvoro et al

$\mathrm{mmol} / \mathrm{mL}$ Gadovist or $20 \mathrm{~mL}$ of $0.5 \mathrm{mmol} / \mathrm{mL}$ Omniscan ) over a period of $85 \mathrm{~s}$, collecting thirty-four volumes of 15 axial slices using the same FOV, acquisition matrix and slice locations as the DTI data, but with a TE of $30 \mathrm{~ms}$ and TR of 2.5 seconds. Spectroscopy was performed with chemical shift imaging (CSI) by placing a large slab volume of interest (VOI) measuring 10mm thick, divided to individual grid squares, on the averaged diffusion weighted image (DWI) that showed the maximum extent of the acute stroke lesion, placed to cover the ischemic lesion and contralateral normal brain (Figure 1). The MRS acquisition parameters were: FOV $320 \mathrm{~mm}$, slice thickness $10 \mathrm{~mm}$, acquisition matrix 24×24, TE $145 \mathrm{~ms}$ and TR $1000 \mathrm{~ms}$. Automatic shimming and water suppression were applied. For each phase encoding, 512 complex data points were acquired with a sampling interval of $1 \mathrm{~ms}$.

\section{Image processing}

DTI and PWI. The images were prepared for detailed processing by converting to Analyze format (Mayo Foundation, Rochester, MN, USA) and processing in MATLAB (The MathWorks, Natick, MA, USA). We removed bulk patient motion and eddy currentinduced artefacts from the DTI data by registering the component EPI volumes to the T2weighted volumes acquired as a part of the DTI protocol using FLIRT (www.fmrib.ox.ac.uk/fsl, a three-dimensional computational image alignment program). Maps of DWI and ADC were derived from the DTI data. Cerebral blood volume (CBV, area under concentration/time curve), mean transit time (MTT, first moment of concentration/time curve) and cerebral blood flow (CBF, CBV/MTT) images were calculated from gamma-variates with arterial input function but without deconvolution ${ }^{29}$. The gamma variate functions were fitted on a voxel-by-voxel basis to PWI concentrationtime curves. The PWI data were also coregistered to T2/DWI data. 
JNNP 2008/168393 revision 3, Cvoro et al

Spectroscopic data were interpolated to a $32 \times 32$ matrix, and $10 \mathrm{~mm}^{3}$ voxels, followed by zero-order phase correction performed on a voxel-by-voxel basis using the residual water signal for each voxel. This "self-referencing" scheme corrects for eddy current effects and brings water on resonance in each voxel. We assigned a fixed chemical shift of 4.70ppm to the water resonance. Residual water signal was removed using Hanckel-Lanczos singular value decomposition ${ }^{30}$. Following Fourier transformation, the spectra were modelled by five Gaussian peaks (corresponding to choline, creatine, NAA containing compounds and lactate) using the AMARES algorithm ${ }^{31 ; 31}$ within the MRUI. ${ }^{32}$. Spectra were automatically discarded if fitted line widths were less than $1 \mathrm{~Hz}$ or greater than $10 \mathrm{~Hz}$, if the metabolite peaks were more than $0.1 \mathrm{ppm}$ offset from their expected position, if the voxels lay on the edges of the PRESS excitation region, if the spectra were of poor quality, fell outside brain parenchyma or contained $>25 \%$ CSF. Metabolite peak areas (in 'institutional' units) were standardised and corrected for scanner drift with monthly spectroscopy phantom quality assurance data.

Tissue voxel classification: We classified the voxel appearance on the diffusion image by applying a grid with voxels of dimension $4.7 \times 4.7 \mathrm{~mm}$ over the entire DWI slice corresponding to the MRS slice position (Figure 1). We used this smaller voxel grid applied to the DWI image because smaller voxels on the DWI image allowed more precise and reproducible classification of individual voxels than did larger voxels (equivalent in size to the CSI grid) in pilot testing. The DWI images were all windowed on the same standard signal intensity to optimize signal contrast between normal and abnormal tissue. A neuroradiologist classified each voxel as: 'definitely abnormal', 'possibly abnormal', or normal ('ipsilateral' or 'contralateral') according to the DWI appearance, blind to all other data. We used this operational voxel classification ${ }^{33}$ to avoid introducing bias that might occur by using ADC or PWI thresholds that so far have not been shown to discriminate 
JNNP 2008/168393 revision 3, Cvoro et al

between viable and non-viable tissue, ${ }^{2 ; 4}$ because DWI signal intensity (not ADC) was the parameter which correlated most closely with cell death or viability in experimental models in comparison with histology, ${ }^{34}$ and because the DWI appearance is immediately visible and rapidly assessed in the acute stroke situation (all other parameters including ADC values require some image processing). Voxels falling outside brain parenchyma were excluded. 'Definitely abnormal' voxels contained clearly hyperintense (bright white) tissue; 'possibly abnormal' voxels contained tissue which appeared subtly hyperintense relative to normal tissue (i.e. in between background normal tissue and hyperintense 'definitely' abnormal tissue); 'normal' voxels contained normal-appearing tissue (Figure 1). Mismatch was defined as voxels which fell within MTT-abnormal, DWI-normal tissue. We tested inter-rater reliability of classifying voxels. The classification based on DWI is the most reproducible, with limits of agreement of the DWI signal values of less than +/$10 \%$. This is in accordance with the experimental literature and probably occurs because the variation in DWI signal is less, with narrower standard deviations than is the case with either ADC or PWI values. The DWI voxel classification was interpolated to the MRS voxel grid (size $10 \times 10 \mathrm{~mm}$ ). The MRS voxels were weighted by the proportion of each DWI-coded tissue present. The DWI voxels interpolated very well to the MRS voxels as the majority contained tissue of only one classification. Note that because the coded DWI/PWI slice $(5 \mathrm{~mm})$ was positioned through the centre of the CSI slice, ie so that $2.5 \mathrm{~mm}$ of the $10 \mathrm{~mm}$ thick CSI slice overlapped on either side of the DWI/PWI slice, the coded slice captured the most reliable CSI information. Note that with the addition of the $1 \mathrm{~mm}$ slice gap on either side of the DWI slice (hence total thickness $7 \mathrm{~mm}$ ), there would only be $1.5 \mathrm{~mm}$ of the DWI slices above and below the CSI slice actually overlapping with the CSI slice, leaving the remaining $4.5 \mathrm{~mm}$ of these adjacent slices uncovered by and not corresponding to the CSI slab. Hence, coding the DWI slices on either side of the index 
JNNP 2008/168393 revision 3, Cvoro et al

slice and attempting to interpolate the small proportion of the DWI slice that overlapped the CSI slab into the CSI grid would not be valid. Furthermore, spectroscopy is very sensitive to partial volume effects at the margins of the CSI acquisition, so any DWI classification from the slices immediately above and below the central DWI slice would be prone to greater error in the metabolites. However, we checked the DWI slices on either side of the index slice to make sure that the voxel coding was similar. Metabolites (in absolute "institutional units"), perfusion (CBF, CBV and MTT, expressed as "index units" normalised to the contralateral normal hemisphere) and diffusion (ADC) values (expressed as absolute values) were extracted from each voxel type to allow an analysis by absolute metabolite as well as relative (to the contralateral hemisphere) values.

\section{Statistical analysis}

All statistical tests were performed in SPSS 14.0 for Windows (SPSS Inc, Chicago, Ill, USA). Metabolite, ADC and PWI values were not normally distributed so we used nonparametric Wilcoxon Signed Rank Sum Tests to compare the metabolite concentrations, ADC and PWI parameters between different voxel classes. We compared metabolites, DWI and PWI parameters within each voxel class using the Spearman Rank Correlation coefficient $(\rho)$, setting significance at the 0.05 level (2-tailed). We examined the relationship between imaging parameters, stroke severity (NIHSS score) and time from stroke to imaging on a per patient basis. We tested associations with both absolute and relative metabolite values to account for any age-related changes in normal tissue. We examined the subgroup of patients imaged within six hours of stroke. Finally we combined 'definitely' and 'possibly abnormal' tissue to create an 'abnormal' category and performed general linear regression to test for independent associations between metabolites, diffusion and perfusion parameters in the DWI normal versus abnormal appearing tissue. 
JNNP 2008/168393 revision 3, Cvoro et al

\section{RESULTS}

\section{Patient characteristics}

We recruited 53 patients with ischaemic stroke; three patients were unable to complete the full imaging protocol. Of the remaining 50 patients with ischemic stroke and complete imaging, four with no visible DWI lesion, and three with poor quality spectra (due to movement) were excluded leaving 43 patients for the present analyses.

The 43 patients had a median age of 76 years (range 37-95, SD 11). The time from stroke to imaging ranged from 1.5-24 hours (median 8, SD 6.6 hours): 14 (32\%) patients were scanned within six hours, 14 (32\%) between six and 12 hours, and 15 (36\%) patients between 12 and 24 hours after stroke. $24(56 \%)$ patients had a partial anterior circulation stroke (PACS), and 19 (44\%) had a total anterior circulation stroke (TACS). The median NIHSS was 9 (range 1-29, SD 7.8); 22/43 patients (51\%) had DWI/PWI mismatch on MTT. None received thrombolysis or any investigational treatment and none had carotid stenosis of more than $50 \%$.

\section{Association with tissue appearance on DTI}

NAA was lowest in the DWI 'definitely abnormal' voxels, and lower in 'definitely' than in 'possibly abnormal' voxels (97.9 vs $113.3, \mathrm{p}=0.01)$, and in 'possibly abnormal' than in 'mismatch' and 'normal' voxels (Table 1; Figure2). There was no difference in NAA between mismatch and normal tissue. That is, NAA was reduced only in tissue that appeared in any way abnormal on DWI; the more abnormal the tissue appeared, the lower the NAA, and therefore the greater the neuronal loss. 
JNNP 2008/168393 revision 3, Cvoro et al

Lactate was highest in 'definitely abnormal' voxels, and successively lower in 'possibly abnormal', 'mismatch', 'ipsilateral' and 'contralateral normal' tissue (all comparisons $\mathrm{p}<0.01)$.

There was no difference in ADC between 'definitely' and 'possibly abnormal' voxels, but ADC was reduced in DWI abnormal compared with 'mismatch' and 'normal' voxels (Table 1; Figure2).

There was no difference in CBF or MTT values between 'definitely' and 'possibly abnormal' voxels. CBF was reduced and MTT prolonged in DWI abnormal compared to 'mismatch' and 'ipsilateral normal' voxels, and MTT was prolonged in ipsilateral compared to 'contralateral normal' voxels.

When the analysis was restricted to the 15 patients imaged within six hours, the significant associations all became non-significant, probably due to the small number of patients $(n=15)$. 
Table 1 Significance of differences between metabolites, DTI and PWI parameters between different voxels classified according to the DWI appearance.

\begin{tabular}{|lcccccc|}
\hline \multicolumn{7}{l}{ Wilcoxon signed rank sum test comparing tissue types } \\
Tissue voxel type & \multicolumn{7}{c}{ (p values) } \\
& ADC & CBF & CBV & MTT & NAA & LACT \\
'definitely' vs 'possibly' abnormal & 0.758 & 0.68 & 0.636 & 0.85 & $\mathbf{0 . 0 1}$ & $\mathbf{0 . 0 0 1}$ \\
'possibly' abnormal vs ipsilateral normal & $\mathbf{0 . 0 0 1}$ & $\mathbf{0 . 0 0 3}$ & 0.06 & $\mathbf{0 . 0 0 6}$ & $\mathbf{0 . 0 0 7}$ & $\mathbf{0 . 0 0 0 1}$ \\
ipsilateral normal vs contralateral normal & 0.57 & 0.32 & 0.05 & $\mathbf{0 . 0 0 0 1}$ & $\mathbf{0 . 0 1}$ & $\mathbf{0 . 0 0 6}$ \\
mismatch vs 'definitely' abnormal & 0.136 & 0.435 & 0.86 & 0.177 & $\mathbf{0 . 0 0 4}$ & $\mathbf{0 . 0 0 2}$ \\
mismatch vs 'possibly' abnormal & 0.088 & 0.095 & 0.263 & 0.291 & $\mathbf{0 . 0 3 9}$ & $\mathbf{0 . 0 1 1}$ \\
mismatch vs contralateral normal & 0.178 & $\mathbf{0 . 0 0 5}$ & 0.158 & $\mathbf{0 . 0 0 0 1}$ & 0.178 & $\mathbf{0 . 0 0 3}$ \\
\hline
\end{tabular}

Wilcoxon signed rank test. (* bold - statistically significant value). 
JNNP 2008/168393 revision 3, Cvoro et al

\section{Associations with time to imaging and stroke severity}

NAA was lower in patients scanned at later times in 'definitely' and 'possibly abnormal' voxels $(\rho=-0.4, p=0.03$ and $\rho=-0.44, p=0.04$ in 'definitely' and 'possibly abnormal' tissue respectively). Lactate, ADC and MTT were not associated with time to scanning in any voxel types. Higher $\mathrm{CBF}$ and $\mathrm{CBV}$ values were associated with increasing time from onset to scanning only in possibly abnormal tissue $(\rho=0.52$, $\mathrm{p}=0.0001, \rho=0.4, \mathrm{p}=0.008$ respectively). When restricted to the 15 patients imaged within six hours, the significant associations became non-significant, probably due to the small number of patients $(n=15)$. There was no association between NAA or lactate and stroke severity (NIHSS) in any of the voxel types. More severe stroke was associated only with lower ADC and CBF in 'possibly' abnormal voxels $(\rho=-0.5$, $\mathrm{p}=0.003, \rho=-0.32, \mathrm{p}=0.04$ for ADC and CBF respectively) and with prolonged MTT in 'ipsilateral normal' voxels only $(\rho=0.6, p=0.001)$. When restricted to the 15 patients imaged within six hours, the association between NAA, lactate and stroke severity remained non-significant; $\mathrm{ADC}$ and $\mathrm{CBF}$ in 'possibly abnormal' tissue lost the significant association with NIHSS; and the association between CBF, CBV, MTT and NIHSS in 'ipsilateral normal' tissue became significant. These results should be interpreted with caution due to the small number of patients (15) in this subgroup analysis.

\section{Associations between metabolites and diffusion and perfusion parameters}

Univariate analyses showed no association between NAA and ADC or PWI parameters in DWI 'definitely' (NAA vs. ADC, $\rho=-0.16, p=0.4$; and vs. MTT $\rho=0.33, \mathrm{p}=0.054$ ) or 'possibly' (NAA vs. ADC, $\rho=-0.24, \mathrm{p}=0.1$; and vs. MTT 
JNNP 2008/168393 revision 3, Cvoro et al

$\rho=0.172, p=0.28$ ) abnormal voxels. Elevated lactate was associated with reduced ADC and prolonged MTT in 'definitely' (lactate vs. ADC, $\rho=-0.41, p=0.02$; and vs. MTT $\rho=0.42, p=0.02$ ) and 'possibly' (lactate vs ADC $\rho=-0.32, p=0.04$ and vs. MTT $\rho=0.37, p=0.02$ ) abnormal voxels. We repeated the analysis in the 15 patients imaged within six hours. The association between NAA, ADC and PI parameters remained negative and the associations between elevated lactate and reduced ADC/prolonged MTT were also unchanged.

Multivariate modelling confirmed the association between reduced NAA and increasing time to scanning in the whole patient group (Coefficient B -2.4, p=0.001), and between high lactate and reduced ADC (Coefficient B -0.07, p=0.001), but not between any other variables. Restricting the analysis to the 15 patients imaged within six hours did not change these results. 
JNNP 2008/168393 revision 3, Cvoro et al

\section{DISCUSSION}

This study demonstrates several important points concerning characterisation of tissue damage with imaging in acute ischemic stroke. First, the ADC, PWI and lactate values are sensitive to the presence of ischemia (significant differences between DWIabnormal, mismatch and normal tissue) but the absence of any correlation with NAA indicates that they are less specific for cumulative permanent neuronal damage. Second, the elevated lactate but normal NAA in 'mismatch' tissue is consistent with the hypothesis that mismatch tissue is ischemic but still viable. Third, the NAA temporal profile was different to that of $\mathrm{ADC}$, PWI and lactate values - NAA declined with time after stroke, consistent with experimental models, ${ }^{18}$ whereas ADC, PWI and lactate values showed no clear temporal profile. Fourth, the acute ischemic lesion appearance on DWI (the "whiteness"), but not the ADC value, corresponds with the degree of neuronal damage, consistent with experimental data where DWI signal intensity but not ADC level was associated with histologically-determined neuronal damage. ${ }^{34}$ Thus in the acute ischemic lesion, the lack of association between NAA and ADC, MTT and lactate, may explain the lack of any consistent ADC or PWI threshold identified to date to distinguish salvageable from non-salvageable tissue. $^{2 ; 4}$

The strengths of the present study include the large sample size (for a complex stroke imaging study), careful image registration and detailed voxel-based analysis. The use of DWI appearance in the image analysis means that the results are independent of any threshold values and also directly translatable to clinical practice, fast and immediate to apply - the brighter the DWI signal, the greater the neuronal damage, and presumably the less likely that the tissue will be salvageable (but this would 
JNNP 2008/168393 revision 3, Cvoro et al

require further testing). We could have used a ADC or PWI threshold to delineate the acute stroke lesion, but no consistent threshold has yet been identified that reliably identifies salvageable/non-salvageable tissue. ${ }^{4}$ Voxel-based analysis, although much more detailed than region-of-interest approaches, may suffer from partial volume effects at lesion edges. We were very careful to exclude poorly fitted or noisecontaminated spectra and all CSF-contaminated voxels. We included patients imaged up to 24 hours after stroke (in prespecified time windows) so as to be able to examine the temporal profile and because there is evidence of substantial amounts of ischemic but viable tissue (50\% of lesion) up to at least 24 hours after stroke. ${ }^{35}$ Whether or not this apparently viable tissue is salvageable with revascularisation therapies beyond six hours is currently uncertain and the subject of ongoing trials. When we restricted analysis to just those patients imaged within six hours of stroke, the results in general did not change although many of the significant associations lost their significance, most likely due to the small sample size.

The study also has some limitations. Lack of out-of-hours scanner availability hampered recruitment (although there is no evidence that patients admitted during the night were different to those seen during the day). Not all patients were able to complete the scanning protocol, particularly those with severe stroke, so some patients that started imaging did not contribute to the analysis. We used a single thick spectroscopic imaging slab centred on the DWI slice that showed the acute stroke lesion at its most extensive; the spectroscopy slice covered the entire thickness of the DWI and PWI slice that showed the maximum lesion extent, plus $2.5 \mathrm{~mm}$ on either side, thereby capturing representative spectroscopic information across a large proportion of the stroke lesion. We tested ways of including the DWI/PWI slices on 
JNNP 2008/168393 revision 3, Cvoro et al

either side of the central DWI/PWI slice in the analysis, but found that this would introduce more error due to lack of overlap of the additional DWI/PWI slices with the CSI slice and increased risk of partial volume averaging. During protocol development, we also tested an alternative of placing the CSI slab with one edge starting with the top edge of a DWI/PWI slice so that the CSI slab then covered one DWI/PWI slice plus a slice gap plus 4mm of the next DWI/PWI slice but this resulted in more problems than it helped due to difficulty in averaging the DWI tissue classification of two adjacent slices except in the very largest lesions. We have tried various other ways of matching the CSI to the DWI/PWI by varying slice thickness and gaps but the constraints of slice number and need for brain coverage mean that there really is no better way of dealing with this problem. The thickness of the CSI slab is limited by signal to noise - too thin a slab results in very poor signal to noise. The MRS slab provided much more information than the single voxel MRS used in most previous studies. Acquisition of multiple adjacent spectroscopy slices would be too time-consuming to be feasible in acute stroke. We did not use metabolite ratios because their use is questionable as there is no reliable denominator that does not change in acute ischaemic stroke: both choline and creatine are altered by the ischaemic process. ${ }^{36}$ In spite of these limitations, many previous studies of brain proton magnetic resonance spectroscopy (MRS) in ischaemic stroke expressed their data as metabolic ratios, mostly to choline or creatine. As it is now known to be incorrect to assume that choline and creatine remain constant in acute ischaemic stroke, ${ }^{36}$ this practice should probably cease. Some variations in alternative metabolite ratio denominators may be interesting, for example the lactate/NAA ratio which is a sensitive metabolic index of acute ischaemia which was used to demonstrate the wide metabolic heterogeneity inside the abnormal area delineated by ADC map. ${ }^{24}$ Indeed, 
JNNP 2008/168393 revision 3, Cvoro et al

the increasing lactate level combined with the simultaneous decreasing NAA level may enhance the diagnostic value of this metabolic ratio and avoid potential problems due to increased water content from cellular oedema. ${ }^{24}$ It is difficult to perform absolute MRS quantification of brain metabolites due to variations in water content arising from brain pathology. The additional sequences (absolute T1 quantification) required to overcome this would make the scan times intolerable for acutely ill patients and as the tissue water increase in the first 24 hours of stroke is less than $6 \%$ it would seem reasonable to use standardised individual metabolite values. The correlations of metabolites with time after stroke should be interpreted cautiously because these data come from different patients imaged at different times after stroke, not the same patients imaged serially. The data on patients imaged within six hours should also be interpreted cautiously as the sample size (15 patients) is small.

One previous study of six patients found a strong correlation between lesion ADC and NAA and lactate ${ }^{24}$ using a region of interest rather than voxel based approach. In a previous region of interest analysis in the same patient population, ${ }^{37}$ we found an association between the ratio [lesion $\mathrm{NAA}] /[$ lesion $\mathrm{NAA}+$ choline+creatine] and reduced ADC (Spearman $\rho=0.434, \mathrm{p}=0.005$ ) but noted that this composite ratio is difficult to interpret given that neither NAA nor choline are constant in acute stroke. ${ }^{36}$ Therefore we did not repeat the analysis with that ratio using the grid-based analysis. Also in agreement with Nicolai et al, in both the present analysis and the previous region of interest analysis, ${ }^{37}$ we have shown an association between rising lactate and falling ADC. Nicolai et $\mathrm{al}^{24}$ also commented on the pronounced heterogeneity of ADC within the lesion which we have highlighted as a finding in the present analysis. The association of elevated lactate with both falling ADC and prolonged MTT in the 
JNNP 2008/168393 revision 3, Cvoro et al

present study means that we would most likely have found a similar association to that of Nicolai $^{24}$ for the ratio of lactate/NAA with ADC and MTT. Another study (14 patients) found no correlation between ADC and NAA in the first 24 hours. ${ }^{16}$ The lack of association between ADC and NAA could be explained by different rates of change. ADC falls immediately after stroke ${ }^{38}$ whilst NAA represents cumulative neuronal death and levels decline more gradually. ${ }^{17-19}$

The presence of lactate in normal appearing tissue on T2-weighted imaging has been reported previously in 11 patients between 2-24 hours after stroke. However, as T2weighted imaging is less sensitive to ischemic change than DWI, it is possible that some of the normal appearing tissue on T2-weighted imaging was actually ischemic. ${ }^{39}$ Another study found lactate outside the diffusion (ADC) lesion. ${ }^{22}$ In both studies the lactate could have been in mismatch tissue. Some lactate in DWI normal tissue ipsilateral to the stroke could be accounted for by partial volume effects from adjacent mismatch voxels but we also found lactate in contralateral normal brain. This could be an artefact of fitting lipids, or contamination from lactate entering CSF from ischemic brain in patients with significant cerebral atrophy. However, the most likely reason is that lactate can be found in healthy brain in older people. ${ }^{40}$

NAA is found exclusively in functioning neurons. ${ }^{14 ; 15}$ Several ischemia models demonstrated an initial rapid decrease of NAA following induction of ischemia, then a further slower decline ${ }^{13}$ that correlated closely with histological evidence of neuronal death. ${ }^{18 ; 19}$ The NAA had fallen to $50 \%$ of normal by 6 hours and to $20 \%$ of normal by 24 hours, corresponding with similar proportions of non-viable neurons identified histologically. ${ }^{18}$ Although accumulation of oedema fluid in the ischaemic lesion could 
JNNP 2008/168393 revision 3, Cvoro et al

"dilute" the NAA and account for some of the apparent reduction in NAA, generally within the first 24 hours the increase in water content is around $6 \%$ as judged by experimental and patient data, ${ }^{41}$ which would not be enough to account for the magnitude of metabolite changes that we have detected. Thus it is reasonable to regard NAA reduction as specific for neuronal loss in acute ischemia. In contrast, at subacute times, there is some uncertainty about whether any residual NAA detected spectroscopically represents a few still viable neurons, neuronal debris, infiltrating microglia, or possibly migrating neural stem cells. ${ }^{14}$ This pattern of early NAA loss has been confirmed in patients. ${ }^{17 ; 42}$ In contrast, in animal models, the ADC value falls as neuronal and glial swelling develops, and then either remains low in persistent occlusion models until dead cells of all types are lysed, or rises in transient ischemia models due primarily to resolution of glial swelling. ${ }^{34}$ Indeed histological comparisons suggest that the ADC is a better marker of glial cell status than of neuronal viability, with the DWI signal ("whiteness") being a better marker of neuronal death than the ADC, ${ }^{34}$ which is why we used the DWI lesion brightness to classify the voxels. The lack of specificity of ADC values for neuronal death is also suggested by the wide range of values found in definite infarcts. ${ }^{43}$ These results may explain the lack of any clear ADC or PWI threshold for reliably differentiating permanently damaged from still viable ischemic tissue. 
JNNP 2008/168393 revision 3, Cvoro et al

Acknowledgements: This study was funded by the Scottish Executive's Chief Scientist Office (Reference \# C2B/4/14), the Row Fogo Charitable Trust (Dr Armitage), the UK Stroke Association (ref TSA 02/01, Dr Cvoro), the Royal Society of Edinburgh/Lloyds TSB Foundation (Dr Rivers studentship) and the Cohen Charitable Trust (Dr Carpenter). JMW is part funded by the SINAPSE collaboration (Scottish Imaging Network. A Platform for Scientific Excellence, www.sinapse.ac.uk) which is funded through the Scottish Funding Council. The work was conducted at the SFC Brain Imaging Research Centre (www.sbirc.ed.ac.uk/), a centre in the SINAPSE Collaboration (www.sinapse.ac.uk).

Disclosure/Conflict of Interest: The authors have no conflicts of interest.

License: The Corresponding Author has the right to grant on behalf of all authors and does grant on behalf of all authors, an exclusive license (or non exclusive for government employees) on a worldwide basis to the BMJ Publishing Group Ltd and its Licensees to permit this article (if accepted) to be published in the Journal of Neurology, Neurosurgery \& Psychiatry editions and any other BMJPGL products to exploit all subsidiary rights, as set out in our license (http://jnnp .bmjjournals.com/ifora/licence.pdf). 
JNNP 2008/168393 revision 3, Cvoro et al

References

1. Sobesky J, Zaro WO, Lehnhardt FG, Hesselmann V, Neveling M, Jacobs A, Heiss WD. Does the mismatch match the penumbra? Magnetic resonance imaging and positron emission tomography in early ischemic stroke. Stroke 2005;36:980-5.

2. Fiehler J. ADC and metabolites in stroke: even more confusion about diffusion? Stroke 2003;34:6-7.

3. Kane I, Sandercock P, Wardlaw J. Magnetic resonance perfusion diffusion mismatch and thrombolysis in acute ischaemic stroke: a systematic review of the evidence to date. J Neurol Neurosurg Psychiatry 2007;78:485-91.

4. Bandera E, Botteri M, Minelli C, Sutton A, Abrams KR, Latronico N. Cerebral blood flow threshold of ischemic penumbra and infarct core in acute ischemic stroke. A systematic review. Stroke 2006;37:1334-9.

5. Kane I, Carpenter T, Chappell F, Rivers C, Armitage P, Sandercock P, Wardlaw J. Comparison of 10 different magnetic resonance perfusion imaging processing methods in acute ischemic stroke. Effect on lesion size, proportion of patients with diffusion/perfusion mismatch, clinical scores, and radiologic outcomes. Stroke 2007;38:3158-64.

6. Sobesky J, Weber OZ, Lehnhardt FG, Hesselmann V, Thiel A, Dohmen C, Jacobs A, Neveling M, Heiss WD. Which time-to-peak threshold best identifies penumbral flow? A comparison of perfusion-weighted magnetic resonance imaging and positron emission tomography in acute ischemic stroke. Stroke 2004;35:2843-7.

7. Heiss WD, Sobesky J, Hesselmann V. Identifying thresholds for penumbra and irreversible tissue damage. Stroke 2004;35:2671-4. 
JNNP 2008/168393 revision 3, Cvoro et al

8. Fiehler J, Foth M, Kucinski T, Knab R, von Bezold M, Weiller C, Zeumer H, Rother J. Severe ADC decreases do not predict irreversible tissue damage in humans. Stroke 2002;33:79-86.

9. Loh PS, Butcher KS, Parsons MW, MacGregor L, Desmond PM, Tress BM, Davis SM. Apparent diffusion coefficient thresholds do not predict the response to acute stroke thrombolysis. Stroke 2005;36:2626-31.

10. Na DG, Thijs VN, Albers GW, Moseley ME, Marks MP. Diffusion-weighted MR imaging in acute ischemia: value of apparent diffusion coefficient and signal intensity thresholds in predicting tissue at risk and final infarct size. Am J Neuroradiol 2004;25:1331-6.

11. Guadagno JV, Warburton EA, Jones PS, Fryer TD, Day DJ, Gillard JH, Carpenter TA, Aigbirhio FI, Price CJ, Baron JC. The diffusion-weighted lesion in acute stroke: heterogeneous patterns of flow/metabolism uncoupling as assessed by quantitative positron emission tomography. Cerebrovasc Dis 2005;19:239-46.

12. Kidwell CS, Saver JL, Mattiello J, Starkman S, Vinuela F, Duckwiler G, Pierre Gobin Y, Jahan R, Vespa P, Kalafut M, Alger JR. Thrombolytic reversal of acute human cerebral ischemic injury shown by diffusion/perfusion magnetic resonance imaging. Ann Neurol 2000;47:462-9.

13. Monsein LH, Mathews VP, Barker PB, Pardo CA, Blackband SJ, Whitlow WD, Wong DF, Bryan RN. Irreversible regional cerebral ischemia: serial MR imaging and proton MR spectroscopy in a non-human primate model. Am J Neuroradiol 2008;14:963-70.

14. Demougeot C, Marie C, Giroud M, Beley A. N-acetylaspartate: a literature review of animal research on brain ischemia. J Neurochem 2004;90:783.

15. Tsai G, Coyle JT. N-acetylaspartate in neuropsychiatric disorders. Prog Neurobiol 1995;46:540. 
JNNP 2008/168393 revision 3, Cvoro et al

16. Labelle M, Khiat A, Durocher A, Boulanger Y. Comparison of metabolite levels and water diffusion between cortical and subcortical strokes as monitored by MRI and MRS. Invest Radiol 2001;36:155-63.

17. Saunders DE, Howe FA, Van Der Boogaart A, McLean MA, Griffiths JR, Brown MM. Continuing ischemic damage after acute middle cerebral artery infarction in humans demonstrated by short-echo proton spectroscopy. Stroke 1995;26:1007-13.

18. Sager TN, Hansen AJ, Laursen H. Correlation between N-acetylaspartate levels and histopathologic changes in cortical infarcts of mice after middle cerebral artery occlusion. J Cereb Blood Flow Metab 2000;20:780-8.

19. Sager TN, Laursen H, Hansen AJ. Changes in $\mathrm{N}$-acetyl-aspartate content during focal and global brain ischemia of the rat. J Cereb Blood Flow Metab 1995;15:639-46.

20. Lemesle M, Walker P, Guy F, D'Athis P, Billiar T, Giroud M, Demougeot C, Lalande A, Baudouin N, Martin D, Brunotte F. Multi-variate analysis predicts clinical outcome 30 days after middle cerebral artery infarction. Acta Neurol Scand 2000;102:11-7.

21. Parsons MW, Li T, Barber PA, Yang Q, Darby DG, Desmond PM, Gerraty, Tress BM RP, Davis SM. Combined 1H MR spectroscopy and diffusionweighted MRI improves the prediction of stroke outcome. Neurology 2000;55:498-505.

22. Stengel A, Neumann-Haefelin T, Singer OC, Neumann-Haefelin C, Zanella FE, Lanfermann H, Pilatus U. Multiple spin-echo spectroscopic imaging for rapid quantitative assessment of $\mathrm{N}$-acetylaspartate and lactate in acute stroke. Magn Reson Med 2004;52:228-38.

23. Liu Y-J, Chen C-Y, Chung H-W, Huang I-J, Lee C-S, Chin S-C, Liou M. Neuronal damage after ischemic injury in the middle cerebral arterial territory: 
JNNP 2008/168393 revision 3, Cvoro et al

deep watershed versus territorial infarction at MR perfusion and spectroscopic imaging. Radiology 2003;229:366-74.

24. Nicoli F, Lefur Y, Denis B, Ranjeva JP, Confort-Gouny S, Cozzone PJ. Metabolic counterpart of decreased apparent diffusion coefficient during hyperacute ischemic stroke. A brain proton magnetic resonance spectroscopic imaging study. Stroke 2003;34:e82-e88.

25. Parsons MW, Barber PA, Desmond PM, Baird TA, Darby DG, Byrnes G, Tress BM, Davis SM. Acute hyperglycemia adversely affects stroke outcome: a magnetic resonance imaging and spectroscopy study. Ann Neurol 2002;52:20-8.

26. Rumpel H, Khoo JB, Chang HM, Lim WE, Chen C, Wong MC, Tan KP. Correlation of the apparent diffusion coefficient and the creatine level in early ischemic stroke: a comparison of different patterns by magnetic resonance. $J$ Magn Reson Imaging 2001;13:335-43.

27. Walker PM, Ben Salem D, Lalande A, Giroud M, Brunotte F. Time course of NAA T2 and $\mathrm{ADC}(\mathrm{w})$ in ischaemic stroke patients: $1 \mathrm{H}$ MRS imaging and diffusion-weighted MRI. J Neurol Sci 2004;220:23-8.

28. Bamford J, Sandercock P, Dennis M, Burn J, Warlow C. Classification and natural history of clinically identifiable subtypes of cerebral infarction. Lancet 1991;337:1521-6.

29. Carpenter T, Armitage PA, Bastin ME, Wardlaw JM. DSC perfusion MRI quantification and reduction of systematic errors arising in areas of reduced cerebral blood flow. Magn Reson Med 2006;55:1342-9.

30. van den Boogaart A, van Ormondt D, Pijnappel WWF, De Beer R, Ala-Corpela M. Removal of the water resonance from ${ }^{1} \mathrm{H}$ magnetic resonance spectra. In: McWhirter JG, ed. Mathematics in Signal Processing III. Oxford: Clarendon Press 1994: 175-95. 
JNNP 2008/168393 revision 3, Cvoro et al

31. Vanhamme L, van den BA, Van HS. Improved method for accurate and efficient quantification of MRS data with use of prior knowledge. J Magn Reson 1997;129:35-43.

32. van den Boogaart A, De Beer R, Van Hecke A, Van Huffel P, GraveronDemilly S, van Ormondt D. MRUI: a graphical user interface for accurate routine MRS data analysis. Proceedings of the ESMRMB 13th Annual Meeting, Prague 1996;318.

33. Karaszewski B, Wardlaw JM, Marshall I, Cvoro V, Wartolowska K, Haga K, Armitage P, Bastin ME, Dennis MS. Measurement of brain temperature with magnetic resonance spectroscopy in acute ischaemic stroke. Ann Neurol 2006;60:438-46.

34. Rivers CS, Wardlaw JM. What has diffusion imaging in animals told us about diffusion imaging in patients with ischaemic stroke? Cerebrovasc Dis 2005;19:328-36.

35. Markus R, Reutens DC, Kazui S, Read SJ, Wright PM, Pearce DC, TochonDanguy HJ, Sachinidis JI, Donnan GA. Spontaneous salvage of penumbral tissue improves clinical outcomes as late as $12-48 \mathrm{hr}$ after stroke onset. Stroke 2004;35:295.

36. Muñoz Maniega S, Cvoro V, Armitage PA, Marshall I, Bastin ME, Wardlaw JM. Choline and creatine are not reliable denominators for calculating metabolite ratios in acute ischemic stroke. Stroke 2008;39:2467-9.

37. Cvoro V, Wardlaw J, Marshall I, Armitage P, Rivers C, Bastin ME, Carpenter TK, Wartolowska K, Farrall A, Dennis MS. Associations between diffusion and perfusion parameters, $\mathrm{N}$-acetyl aspartate, and lactate in acute ischemic stroke. Stroke 2009;40:767-72.

38. Kucinski T, Vaterlein O, Glauche V, Fiehler J, Klotz E, Eckert B, Koch C, Rother J, Zeumer H. Correlation of apparent diffusion coefficient and computed tomography density in acute ischemic stroke. Stroke 2002;33:1786-91. 
JNNP 2008/168393 revision 3, Cvoro et al

39. Gillard JH, Barker PB, van Zijl PCM, Bryan N, Oppenheimer SM. Proton MR spectroscopy in acute middle cerebral artery stroke. AJNR Am J Neuroradiol 1996;17:873-86.

40. Sijens PE, Den HT, deLeeuw FE, de Groot JC, Achten E, Heijboer RJ, Hofman A, Breteler MM, Oudkerk M. MR spectroscopy detection of lactate and lipid signals in the brains of healthy elderly people. Eur Radiol 2001;11:1495-501.

41. Dzialowski I, Weber J, Doerfler A, Forsting M, von Kummer R. Brain tissue water uptake after middle cerebral artery occlusion assessed with CT. $J$ Neuroimaging 2004;14:42-8.

42. Gideon P, Sperling B, Arlien-Soborg P, Olsen TS, Henriksen O. Long-term follow-up of cerebral infarction patients with proton magnetic resonance spectroscopy. Stroke 1994;25:967-73.

43. Hand PJ, Wardlaw JM, Rivers CS, Armitage PA, Bastin ME, Lindley RI, Dennis MS. MR diffusion-weighted imaging and outcome prediction after ischemic stroke. Neurology 2006;66:1159-63. 
Figure 1: Classification voxel grid (4.7x4.7mm) overlaid on a DWI image (left).
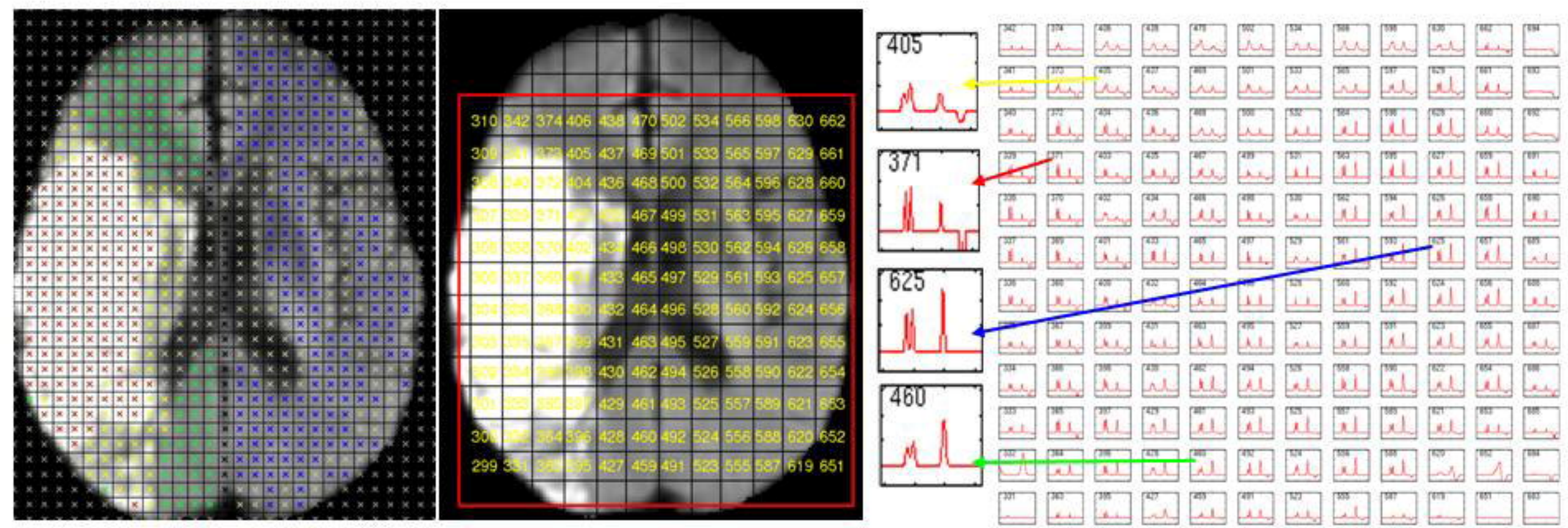

Legend: The classification in each voxel is indicated by a color cross: blue = contralateral normal, green = ip silateral normal, red = 'definitely' abnormal, yellow = 'possibly' abnormal, grey $=\mathrm{CSF}$ or background. This grid was transposed over the larger CSI voxel grid (10 mm3) from which metabolite, perfusion and diffusion data were extracted (center). Representative spectra from each voxel type are shown on the right. 
Figure 2: Box plots of median absolute metabolite (in institutional units), $\mathrm{ADC}$ and PWI values in the ischemic lesion by voxel classification.
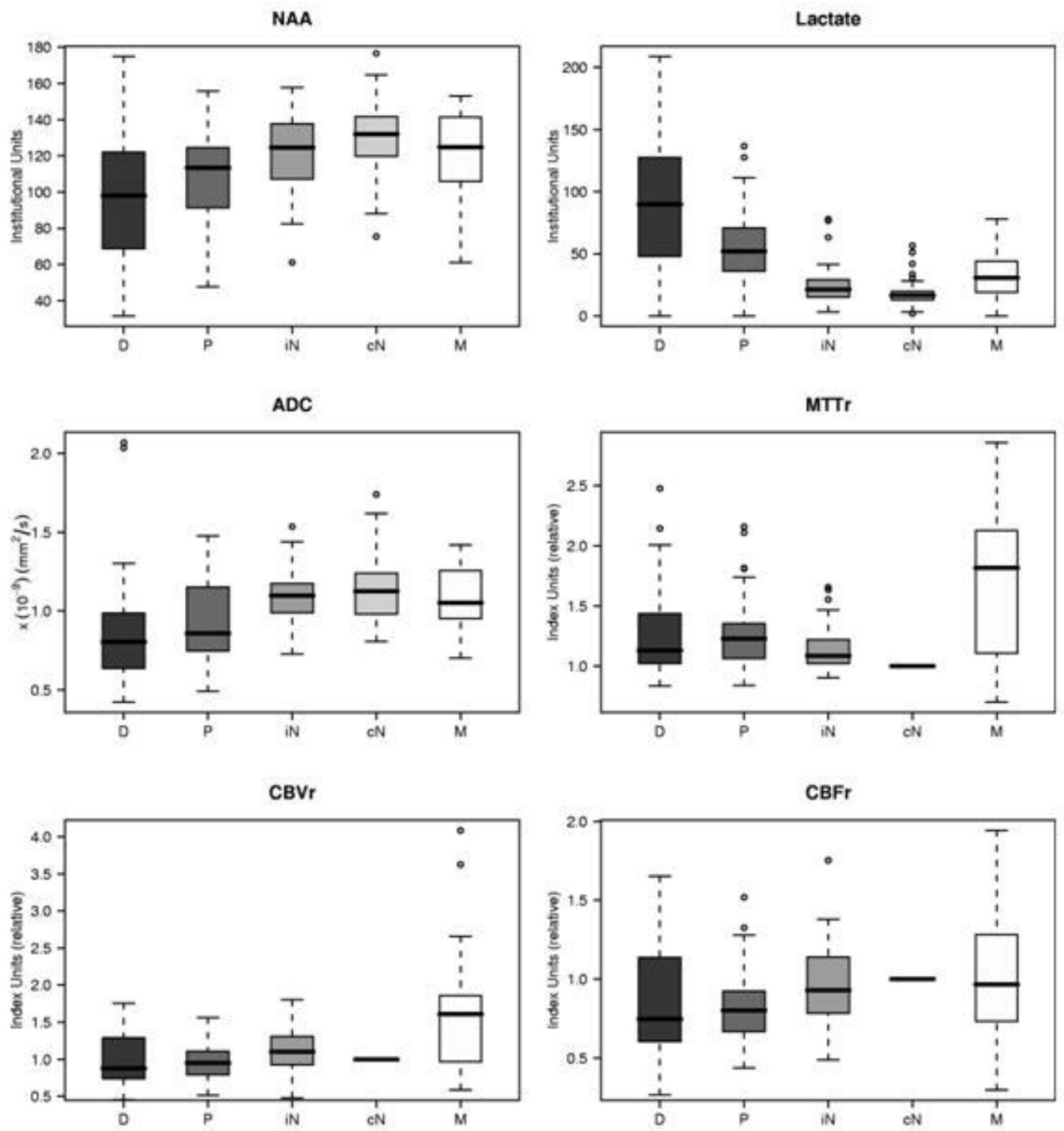

Legend: The box represents the interquartile range; the middle bar represents the median; whiskers represent maximum and minimum values; circles above represent outliers. D, 'definitely' abnormal; P, 'possibly' abnormal; iN, ipsilateral normal; $\mathrm{cN}$, contralateral normal; $\mathrm{M}$, mismatch voxels. 